This item was submitted to Loughborough's Research Repository by the author.

Items in Figshare are protected by copyright, with all rights reserved, unless otherwise indicated.

\title{
The application of digital twin technology in operations and supply chain management: a bibliometric review
}

\section{PLEASE CITE THE PUBLISHED VERSION}

https://doi.org/10.1108/SCM-01-2021-0053

\section{PUBLISHER}

Emerald Publishing Limited

VERSION

AM (Accepted Manuscript)

\section{PUBLISHER STATEMENT}

This paper was accepted for publication in the journal Supply Chain Management and the definitive published version is available at https://doi.org/10.1108/SCM-01-2021-0053.

LICENCE

CC BY-NC 4.0

\section{REPOSITORY RECORD}

Bhandal, Rajinder, Roy Meriton, Richard Kavanagh, and Anthony Brown. 2022. "The Application of Digital Twin Technology in Operations and Supply Chain Management: A Bibliometric Review”. Loughborough University. https://hdl.handle.net/2134/17151485.v1. 
The application of digital twin technology in operations and supply chain management: A

\author{
bibliometric review
}

\begin{abstract}
Purpose

Application of digital twin to optimise operations and supply chain management functions is a bourgeoning practice. Scholars have attempted to keep pace with this development initiating a fast-evolving research agenda. The purpose of this paper is to take stock of the emerging research stream identifying trends and capture the value potential of digital twin to the field of operations and supply chain management.
\end{abstract}

\title{
Design/methodology/approach
}

In this work we employ a bibliometric literature review supported by bibliographic coupling and keyword co-occurrence network analysis to examine current trends in the research field regarding the value-added potential of digital twin in operations and supply chain management.

\section{Findings}

The main findings of this work are the identification of four value clusters and one enabler cluster. Value clusters are comprised of articles that describe how the application of digital twin can enhance supply chain activities at the level of business processes as well as the level of supply chain capabilities. Value clusters of production flow management and product development operate at the business processes level and are maturing communities. The supply chain resilience and risk management value cluster operates at the capability level, it is just emerging, and is positioned at the periphery of the main network. These clusters variously help to shed light on the value-added potential of digital twin in operations and supply chain management

Originality/value

This is the first study that attempts to conceptualise digital twin as a dynamic capability and employs bibliometric and network analysis on the research stream of digital twin in operations and supply chain management to capture evolutionary trends, literature communities and value-creation dynamics in a digital-twin-enabled supply chain.

\section{Keywords}

Digital twin, cyber-physical systems, operations management, supply chain management, bibliometric analysis, bibliographic network analysis, keyword co-occurrence network analysis 


\section{Introduction}

\subsection{Background}

The strategic importance of supply chains has been growing in prominence in recent years with many scholars remarking that increasingly, market competition takes place no longer between individual companies but supply chains (Farahani et al., 2014). Indeed, the rapid evolution of supply chain management (SCM) as a sub-discipline within operations management (OM) provides strong evidence for this (Christopher, 1998). As such, there is widespread recognition of the supply chain as an increasingly important lever of value creation for firms in an ever more dynamic and unpredictable marketplace. The transformation of the operations and supply chain management (OSCM) field into a competitive necessity can be attributed in large parts to successive technological disruptions that have taken place over the years. Digital twin (DT) technology is the most recent instalment of industry 4.0 technologies that promises to further exacerbate the ongoing trend.

The notion of a DT is however not new, it was first introduced back in the 1960s, a practice established and developed by NASA for the Apollo 13 space program. NASA produced physical replicate versions of systems at ground level, which were exact mirror-images of the systems in space. Consequently, this DT technology enabled NASA to simulate and measure situations in the Apollo 13 spacecraft whilst in space (Miskinis, 2019). Thus, with its roots firmly fixed in aeronautics, the DT paradigm is arguably an early example of science and manufacturing fusing together (Barricelli, Casiraghi, and Fogli, 2019).

Although the first vision of a DT dates back more than a decade (Grieves 2002), it has only recently obtained increased research interest within multiple domains. The main reason for 
this lies in the fact that central technological enablers, such as the Internet of Things (IoT), have only recently reached the maturity to be deployed profitably in economic environments. Within the different interested communities, the term has evolved leading to at least two different viewpoints of a DT (Negri et al. 2017). The first defines the DT merely as the simulation of the physical asset itself and is mostly used by engineering scholars. However, beyond the scope of simulation, the second perspective refers to a DT as a model which constitutes the basis for simulations, analyses and the like. The latter perspective is currently the most adopted view on DTs and thus the focused viewpoint of this work (Dietz and Pernul, 2020). In the context of OSCM we thus define a DT as "a virtual replica of the real process operation, which is connected to the real world by sensor data and advanced big data analytical tools" (Verboven et al., 2020, p. 79).

A variety of tools from different areas of technological development are currently being explored for such task. Internet of Things technologies facilitate transfer data from and to different sensors, computers, and machines. Cloud Computing offers ways to store, share and work with the data more effectively (Kavanagh et al., 2015). Data Mining and Artificial Intelligence allow to process the data in a smart and efficient way, more and more like the human brain does (Verboven et al., 2020). Other notable innovations associated with DT include Augmented Reality (AR), Robotics (R), Sensor Technology (ST), Omni Channel (OC), and 3D Printing (3DP), to name a few (Büyüközkan and Göçer, 2018).

These methods and innovations presented above are typically developed in the domain of information and communication technology (ICT) and use software tools for digital data handling, inherent to the strategic initiative Industry 4.0. Therefore, the application of these tools in the supply chain can also be referred to as the digitalization of the supply chain 
(Verboven et al., 2020). The term digital supply chain (DSC) has been coined to reflect the transformative influence of digital technologies such as DT in the operations of supply chain business processes. In fact, the DT has been argued to be a proactive digital approach introducing the next step in SC digitalization (Dietz and Pernul, 2020). A digital supply chain is defined as a smart, value-driven, efficient process to generate new forms of revenue and business value for organisations and to leverage new approaches with novel technological and analytical methods (Sanders and Swink, 2019; Misrudin and Foong, 2019)

DSC is not about whether goods and services are digital or physical, rather, it is about the way in which OSCM processes are managed employing a wide variety of innovative technologies (Büyüközkan and Göçer, 2017). A key value of a DSC is the ability to extract intelligence and optimal decisions from large amounts of real-time data quickly by applying machine learning algorithms to those data streams (Moufaddal et al., 2019). Consequently, under the auspices of IT-enabled supply chain management, the emerging literature concerning the DSC examines how digital technologies such as DT create value in supply chains, and in turn how DSC can add value to firms. Early indications reveal the huge potential of DT to enhance numerous performance metrics at varying levels of OSCM abstractions.

\subsection{Value creation in SCM and DT}

According to Borys and Jemison (1989), value creation is the process whereby the capabilities of partners in a supply chain are combined such that the competitive advantage of one or more of the partners is improved. However, the notion of value has emerged to be much wider than just economic implications (Pal and Sandberg, 2017). Indeed, with the emergence of the new service- and knowledge-based economy, Letaifa (2014) has noticed a shift away from a post-industrial economic understanding of value (cost, efficiency, customer 
expectations) towards a more knowledge based, social, subjective, intangible, and complex perspective. According to a comprehensive review by Schenkel et al. (2015), value manifestations of four types of value, namely economic, environmental, information and customer value, can be identified in the literature.

In the literature, value creation has typically been characterized as either exogenous spanning organisations or endogenous focussing on internal efforts to improve supply chain performance (Jayaram at al., 2004). At the empirical level, research has tended to focus on the intra-and-extra organisational generative mechanisms of value (Meriton et al., 2020) and in establishing a link between those and value manifestations. It is also worth noting however that the supply chain makes up a quasi-organisation where interdependencies, time, and shifting boundaries imposes on firms the imperative of efficient management of relationships of supply chain actors to successfully achieve value objectives of the focal firm.

In this context, one of the key research strands in the supply chain value creation literature focusses on the dynamics of relational capital (Cousins et al., 2006) variously operationalised as relationship management (Kähkönen and Lintukangas, 2018), cooperation (Sousa et al., 2012), collaboration (Fu and Piplani, 2004) or co-creation (Letaifa, 2014). Employing a sample of Finnish companies, Kähkönen and Lintukangas (2018) show that supplier relationship management (SRM) capabilities can be a supply management factor that significantly influences its ability to create value. Sousa et al., (2012) show that greater levels of cooperation with customers have a significant impact on the intensity of lean production adoption, which is considered an important value creation lever. Employing a series of numerical experiments, Fu and Piplani (2004) show that the supply-side collaboration can improve the supply chain performance in terms of better stabilizing effect and service level. 
Research shows the important enabling role of IT, or rather IT capabilities, in enabling and maintaining relational capital in supply chains. IT capability is defined as technological capability used to acquire, process, and transmit information to support organisational decision-making (Grover and Malhotra, 1999) and to facilitate communication, coordination, and collaboration between multiple parties along the supply chain. Capability here emphasises the fact that technology is not inherently valuable, rather, value is incumbent on how it can be exploited to transform SC operations and relationships (Wu et al. 2006). In the research conducted by Fawcett et al. (2011), the authors draw on the resource-based view (RBV) paradigm to describe how IT capabilities could be leveraged to create a higher order collaboration capability. The dynamic collaboration capability is shown to have a positive influence on operational performance and customer satisfaction. An important take-away from this study besides the obvious concerns the ontological status of value in supply chains. It can be observed that value manifests at different levels of abstraction, in other words value could be argued to have emergent properties embedded in processes, capabilities and performance outcomes.

More recent examples of IT-enabled value creation in supply chains can be found in the literature concerning the intersection of big data analytics (BDA) and supply chain management. An interesting study to this effect by Chen and Preston (2015) conceptualises the use of BDA as a dynamic capability. The notion of dynamic capabilities describes how firms integrate, build, and reconfigure internal and external competences to address rapidly and unpredictably changing environments (Teece et al., 1997). The authors reason that BDA can be considered as a dynamic capability due to its organisational information processing capability that reduces uncertainty by stimulating insights and knowledge creation and increases organisational capability for strategic decision. Against this backdrop and employing 
a survey approach the authors show that the use of BDA in supply chains can indeed lead to value creation measured as asset productivity and business growth.

Here we wish to argue that DTs can be viewed through the same lens as an IT capability given the above definition of the latter. Furthermore, according to Qi and Tao (2018) value creation based on data and analytics is very similar to that based on the DT. As we have explained above, data and analytics are indeed an enabling part of the DT. As such and drawing on Chen and Preston's (2015) argument in favour of BDA as a dynamic capability we can similarly come to the same conclusion with respect to a DT. Chen and colleague also reinforce our previous articulation with respect to IT-enabled value creation noting that the value contribution of BDA is incumbent on its embeddedness into and assimilation with supply chain processes. This therefore opens up the potential of BDA-enabled value creation across the landscape of SC business processes.

A comprehensive review of BDA-inspired value creation in the supply chain Meriton et al. (2020) has revealed a multilevel framework of value creation across the supply chain. They show that value is a nested concept and can manifest at different levels, such as the business processes level, the supply chain level as well as the firm level. Drawing on Croxton et al.'s (2001) conceptualisation of supply chain management in terms of a series of interrelated business processes, the authors show that big data analytics could be leveraged to enhance business processes such as demand management, order fulfilment, product management, production management, supply management and customer services management. In turn, enhanced processes could lead to capabilities such as agility, flexibility and collaboration at the supply chain level with a knock-on effect on firm performance. In this work, we seek to draw on the notion of supply chain as business processes to examine the dynamics of value 
at the digital twin and supply chain interface. It is our contention that the influence of DT in supply chains could follow a similar trajectory, however, these dynamics are yet to be captured in a structured and systematic way in the academic literature. We specifically survey the state-of-the-art concerning the value creation potential of DT in OSCM. We recognise that this is a fairly nascent sub-field of the DSC literature where most of the potential of DT for value creation may still be unclaimed, however it is growing in importance and stature warranting a synthesis of the emerging knowledge domains. The study discusses the valueadded potential of DT in OSCM to guide researchers and industry experts in organising, conceptualising, and conducting their research on DT of the future.

\subsection{Previous literature reviews}

The fast-developing research stream on the application of digital twin in operations and supply chain context has prompted a growing number of literature reviews. However, most of these reviews are narrative (see Table 1). A systematic literature review is preferred to the traditional approach as it is replicable, scientific, evidence-based, and transparent (Tranfield, Denyer, and Smart, 2003). Earlier reviews on the application of digital twin in operations and supply chain management (DT-OSCM) have focused on enabling technologies $(f=22)$, manufacturing $(f=18)$, supply chain management $(f=4)$, product design $(f=3)$, additive manufacturing $(f=3)$, sustainable business model $(f=1)$ and logistics $(f=1)$. To address this research gap, we present a systematic and bibliometric literature review of the application digital twin technologies in operations and supply chain management (DT-OSCM) based on bibliographic coupling analysis. A total of 1856 research articles, published from 2011 to 2021 were collected, and 234 articles met our inclusion criteria for the review. The main objectives of the review are to (i) identify the major trends in DT-OSCM research (ii) identify the major 
research fronts in DT-OSCM and (iii) identify value potential of DT, and (vi) set a research agenda.

The remainder of this paper is organised as follows. In Section 2, we discuss our research methodology along with some initial statistics regarding recent trends in the output of DTOSCM research. In Section 3 we present our initial bibliometric analysis resulting in additional author and affiliation statistics. Section 4 presents a detailed network analysis including a bibliographic coupling of citing articles and a co-occurrence network analysis of influential keywords which leads to the identification of key communities of primary research streams areas and subsequent discussions in Section 5. We conclude this paper in Section 6 with implications for practice, theory, and future research.

\section{Methodology}

Literature reviews aim to summarize and synthesize earlier studies to illuminate what has been done regarding a particular research problem (Boell and Cecez-Kecmanovic, 2014) and identify potential avenues for further research highlighting the boundaries of existing knowledge (Tranfield et al., 2003). Systematic literature reviews aim to summarize research studies related to a specific research question in a way that is fair, rigorous, and auditable. Systematic reviews are different from traditional narrative reviews in many ways. Narrative reviews tend to be mainly descriptive, lacks a systematic search of the literature and thereby often focus on a subset of studies in an area chosen based on availability or author selection (Uman, 2011). Thus, while informative, narrative reviews, can often include an element of selection bias (Srivastava, et al., 2018). They can also be confusing at times, particularly if similar studies have diverging results and conclusions (Evangelista and Durst, 2015). 
Systematic reviews, as the name suggests, typically involve a detailed and comprehensive plan and search strategy derived a priori, with the goal of reducing bias by identifying, appraising, and synthesizing all relevant studies on a particular topic (Uman, 2011). Rowley and Slack (2004) provide guidance for a structured methodology involving scanning resources, designing the mind map to structure the literature review, writing the study and building the bibliography. Following in their footsteps, we adopt a five-step methodology for data collection and comprehensive evaluation of the field aiming to identify the most influential studies, determine the topical areas of research and provide insights for current research interests and directions for future research in the field.

\subsection{Defining the search terms}

Keywords used for data collection include "Digital Twin", "Virtual Twin", "Digital Shadow", and "Evaluation Twin". To arrive at these keywords, we scour practice-based articles published on the internet as well as academic literature on digital twin to capture the nomenclature employed by authors in discussions on digital twin. While digital and virtual twin are used interchangeably, digital shadow is a closely related concept. A digital shadow is a replica of all processes instantiated within a physical system such as a production system (Ehrhardt and Hoffmann, 2020). While a digital twin can be used for simulations, a digital shadow is exclusively descriptive. The use of a digital shadow promises to offer potential in the application of predictive analytics. Not only is a digital shadow a vital component of a digital twin, but it is also increasingly being discussed as a more cost-effective representation of physical systems without the need of complex simulations. The term evaluation twin has been used to emphasise a digital twin designed to solely perform evaluative tasks (Zhang et 
al., 2020). The list of keywords generated was validated with the help of an expert digital twin practitioner engineer.

\subsection{Initial search results}

Using the "title, abstract, keywords" search in the Scopus database, publication with the keywords discussed above were downloaded and stored. Scopus is now considered the largest searchable citation and abstract source of searching literature which is continually expanded and updated (Rew et al., 2009). Comparisons are often drawn between SCOPUS and Web of Science (WOS) in discussions regarding the most suited database for a particular study. One of the main differences between SCOPUS and WOS databases is the breadth of coverage. In a comparative study Vieira and Gomes (2009) found that Scopus provides $20 \%$ more coverage than WOS. Their results reveal that $2 / 3$ of these records can be found in both databases but $1 / 3$ of the records are only in one database (Aghaei et al., 2013). Furthermore, in a recent systematic review on blockchains application in supply chains, Pournader et al., (2020) decided to retain Scopus as the main search database following comparative searches which yielded Scopus search results that completely covered the WOS search outcomes whilst introducing more academic resources. The initial search in Scopus was conducted on 12 January 2021 and it returned a total of 1856 resources, see Table 2 for details. By further limiting the search to articles and reviews written in English the sample was reduced to 652 . In the next step, two of the authors independently reviewed all the titles and abstracts to ensure that the papers meet with our inclusion criteria. The final lists of included articles were verified by another author and any discrepancies were resolved during an online meeting involving all the authors. The main inclusion criteria was designed to capture the modalities 
of value-creation by digital twins in operations and supply chain management. Thus, during the screening process colleagues were guided by the following criteria:

- Conceptual, empirical and modelling studies on implementation of digital twin at various supply chain levels including, supply chain business processes, emergent supply chain capabilities and other related operations.

- Studies that could be downloaded as full text.

Application of this inclusion criteria produced a final sample of 234 papers which served as the primary data source for the literature analysis. The final agreed sample was stored in RIS format to include all the essential paper information such as paper title, authors' names and affiliations, abstract, keywords and references (Fahimnia et al., 2015).

\section{[Insert Table 2 here]}

\subsection{Initial data analysis}

Figure 1 illustrates the trend in publishing from 2011 to 2021 suggesting DT-OSCM to be a fairly nascent field of study, barely 10 years old. A constant growth in the number of publications can be observed with a particularly significant increment between 2019 and 2020. The initial statistics also show that 99 journals have contributed to the publication of those 234 papers. Those journals appear to represent the engineering and manufacturing fields more substantially. Table 3 shows the contribution of journals in which at least four of the DT-OSCM papers have featured.

[Insert Figure 1 here]

[Insert Table 3 here] 


\section{Data analysis}

There are two parts to data analysis, and these include 'bibliometric analysis' and 'network analysis'. A bibliometric analysis provides summaries of data, which provide a broad perspective on the activities and impact of research, especially in terms of most cited researchers, articles, keywords, countries, and universities (Ferreira et al., 2020). We employ BibExcel for the bibliometric analysis due to its capability to handle large data sets and its compatibility with different computer applications including Excel, Pajek and Gephi (Persson et al., 2009). BibExcel is also used to prepare the input data for a detailed network analysis (Fahiminia et al., 2015). The network analysis part uses an open-source visualisation tool called Gephi to perform topical content-based classification of the existing DT-OSCM research. Gephi is preferred over other existing network analysis software such as Pajek (Batagelj and Mrvar, 2011) and VOSviewer (van Eck and Waltman, 2013) due to its capability to work efficiently with large data sets, and its flexibility to develop a wide range of innovative analysis and investigation options.

\subsection{Bibliometric analysis}

The initial author and affiliation statistics obtained from our initial bibliometric analysis are presented and discussed this section. Identifying the key researchers and universities in different geographical regions can help scholars and students who are interested in conducting research of digital twin in OSCM with researchers from other universities. BibExcel, an open-source bibliometric toolbox was employed to perform the initial bibliometric and statistical analyses and to prepare the raw data for additional network analysis. BibExcel is a tool for analyzing bibliographic data or any data of a textual nature formatted in a similar manner (Persson et al., 2009). A default input file contains data fields 
that can be reformatted into other formats to retrieve bibliometric information. The data output can be exported to Excel or any program that takes tabbed data records. Fahimnia et al. (2015b) observe that this high degree of flexibility makes BibExcel a powerful tool, however, relatively difficult to work with especially in performing the initial setup. RIS format, Scopus output, was used as input for BibExcel.

\subsubsection{Author influence}

We extracted the author field from the Bibexcel doc file and recorded the frequency of appearance of all authors associated with the sample of 234 articles. Most of the contributing authors appeared in only a single paper. Only $18 \%$ of 699 contributing authors have contributed to more than one paper, leaving 576 authors appearing in only one paper. In Table 4, the key contributing authors based on the number of published articles, are listed. We also performed an analysis to capture key collaborating authors. Table 5 shows collaborating authors outcomes with the majority of authors listed in both tables. This is somewhat encouraging suggesting active collaboration between researchers in the early stages in the development of the field. Active collaboration between researchers can have a significant impact on productivity and is critical to the development and establishment of a new knowledge domain.

[Insert Tables 4 and 5 here]

\subsubsection{Affiliation statistics}

The address field containing the affiliations information for the authors were extracted from the RIS file in BibExcel. For each affiliation, the city where the institution is located was 
obtained. The coordinates of these cities were obtained from gpsvisualizer.com to plot the geographical locations of institutions contributing to the literature as shown in Figure 2. Each red circle represents one institution. Greater density of contributing institutions can be found in Europe, Germany in particular, in spite of China boasting the largest number of contributing institutions as a single country. The figure also summarises the top contributing countries.

[Insert Figure 2 here]

The top contributing institutions, their geographical locations and the quantity of published papers are shown in Table 6. The geographical dispersion of these institutions demonstrates that digital twin in OSCM research has attracted institutions from around the globe. Surprisingly, none of the top institutions in Table 5 are located in North America (USA more precisely). The effort to establish a research agenda around digital twin application in OSCM in the US appears still fragmented compared to Europe and parts of Asia. While there is clearly an appetite, evidenced by the large cohort of participating institutions, leading research centres are yet to take root US. This is however an assumption and additional research would be needed to determine the veracity of this initial supposition.

\section{[Insert Table 6 here]}

We performed an additional analysis to capture international collaborations between authors from different institutions. International research collaborations can help elevate an institution's international ranking and reputation and can also boasts its ability to attract research funding. The results displayed in Table 7 show China leading the way, collaborating with 7 other countries on a total of 38 papers followed by the US also collaborating with 7 other nations but on fewer publications. It can also be observed from Table 7 the significant 
contributions of developing countries such as China, Russia, India, Hong Kong and Singapore being active internationally.

[Insert Table 7 here]

\subsection{Network analysis and literature clustering}

A network analysis and graphical investigation followed for the selected sample. "Gephi", an open-source software package for graph and network analysis (Bastian et al., 2009) was employed for this purpose. Gephi uses a 3D render engine to develop illustrations of large networks in real-time and assist in speeding up the exploration process (Gephi, 2013) (Cherven, 2013). The flexible and multi-task architecture facilitates innovative approaches to work with complex data sets and produce insightful visual aids (Fahimnia, 2015b). Gephi provides easy and broad access to network data and assist in specializing, filtering, navigating, manipulating and clustering of data (Bastian et al., 2009). A graph dataset is needed to be generated to enable Gephi to map and visualize literature networks (Fahimnia et al., 2015a) in which published papers are shown as nodes and relationships, such as citations or couplings, are represented by the arcs/edges between the nodes (Grandjean, 2015). The bibliographic data obtained from Scopus (in RIS format) was reformatted into a graph dataset. BibExcel was used to transform the raw data into useable data for Gephi. Gephi accepts various graph data formats including '.NET' that BibExcel can generate.

\subsubsection{Citation analysis}

Different approaches have been employed to examine the significance of a publication. A citation analysis is most commonly employed for this purpose which aims to establish the 
popularity of a publication by establishing the frequency a publication is cited by other publications (Ding and Cronin, 2011). Compared to other approaches such as expert surveys, a citation analysis has an advantage in how it achieves an inter-subjectively testable result as its findings are independent of the subjective viewpoints and opinions of the interviewed experts (Kraus et al., 2012). A further advantage of this approach is that it presents a "real time" state of current research (Tahai and Meyer, 1999). Table 8 shows the top 10 papers based on their number of local and global citations. The local citations reflect the total number of citations an article has received within the sample of 234 articles whereas the global citations provide the overall number of citations in the Scopus database that are related to other research fields.

[Insert Table 8 near here]

\subsubsection{Eigenvector centrality}

"Prestige" is another indicator of importance besides citation counts (Ding et al., 2009). Prestige can be measured as the number of times a paper is cited by other highly cited papers (Fahimnia et al., 2015a). A highly cited paper may not necessarily entail prestige, although in some cases there might be a strong positive correlation between the two (Fahimnia, 2015b). Eigenvector centrality has been argued as a more preferred metric to identify the influential papers in a bibliographic network considering the entire network pattern (Bonacich, 2007; Borgatti, 2005). Eigenvector centrality premises that a node's importance in a network may increase by having connections to the other nodes that are themselves important and it is calculated by giving each node a relative score proportional to the sum of the scores of its neighbours (Bonacich, 2007). Eigenvector centrality $x$ can be defined in two equivalent ways via a matrix equation and a sum as: 


$$
A x=\lambda x \text { and } \lambda x_{c}=\sum_{V=1}^{V} a_{c v} x_{v} \text { respectively, }
$$

where $\lambda$ is the largest eigenvalue of $A$ (Bonacich, 1987). According to Rajagopal et al. (2017) the eigenvector centrality can be large either because a node has many neighbours or because it has important neighbours (or both). This suggests that eigenvector centrality can be treated as a measure of both popularity and importance of a paper in a bibliographic network. Table 9 shows the top 10 papers based on an eigenvector centrality measure. For this study's 125-node network, eigenvector centrality values vary between 0.0011 and 1 . It can be observed that a higher number of global citations cannot guarantee the 'prestige' of a paper in a bibliographic network. In fact, the different rankings produced very different results for our sample.

[Insert Table 9 near here]

\subsubsection{Bibliographic coupling}

Bibliographic coupling was chosen as the network structure to be analysed over others such as co-authoring and co-citation. Two documents are said to be co-cited when they both appear in the reference list of a third document (Egghe and Rousseau, 2002). Small (1973) argues that the frequently co-cited papers represent the key concepts, methods or experiments in a field. Osareh (1996a; 1996b) and Cawkell and Newton (1976) also observe that co-cited papers are significant and related in subject to each other. As such, it has been argued that the clusters of cited documents that make up a co-citation network comprise the intellectual base of the field (Persson, 2010). Bibliographic coupling is premised on the concept that two documents are related if they share the same sets of citations (Small, 1973). In other words, a coupling unit between two documents is an item of reference used by these two documents (Jarneving, 2007). 
Therefore, in bibliographic coupling mapping, clusters of citing papers largely founded on the same intellectual base are produced. Such clusters make up the current research trends (Jarneving, 2005) that comprise of the field of enquiry often representing topical or emerging areas of research (Bornmann and Marx, 2012). Owing to its reliance on cited documents, bibliographic coupling is criticised for being fixed and retrospective, while co-citation analysis is viewed as dynamic and forward looking (Surwase et al., 2012) for its dependence on citing documents. Nevertheless, while facilitating the construction of the intellectual base of the field, co-citation analysis cannot be used to establish current research trends or research authors (Ibekwe - SanJuan, 2009). This led us to capture the dynamics of the fast-evolving DT-OSCM research through the lens of bibliographic coupling which is able to cluster very recent papers (Boyack and Klavans 2010).

The initial bibliographic coupling mapping with Gephi revealed that 159 articles out of a total of 234 have co-cited another paper either internal or external to the sample. A raw network of nodes and edges was generated by the software with a random positioning of the nodes when the '.NET' file was first opened in Gephi. It is difficult to interpret this layout due to a lack of a discernible pattern. The Force Atlas layout (Jacomy, 2009) was then applied to the raw network. The Force Atlas algorithm works in such a way that linked nodes attract and non-linked nodes repulse each other. It also permits the manual adjustment of the repulsion strength, gravity, speed, node size and other characteristics (Bastian et al., 2009). With this algorithm the most connected nodes gravitate towards the centre of the network and the lesser connected nodes away from it.

The Force Atlas layout of the proposed 159-node bibliographic coupling map is shown in Figure 3. Co-citing papers (shown by nodes) are well-connected, least connected nodes are 
positioned more remotely from the centre. To develop meaningful clusters that correspond to a specific theme in the bibliographic network, we first had to filter the network and limit the degree feature of the network to a minimum of 2 to eliminate weakly connected nodes (i.e., papers that were weakly connected to the network due to low co-citing frequency). Excluding weakly connected nodes resulted in a network with 135 nodes and 1634 edges as shown in Figure 4.

\subsubsection{Literature Clustering}

In a bibliographic network each node relates to a given article and the links represent the presence of one or more citations between two publications. The network is represented by the adjacency matrix A of elements aij. The elements aij are set equal to 1 when publication $i$ cites publication $j$ while elements on the principal diagonal (aii) are set equal to 0 , since a publication cannot cite itself (Caschili et al., 2014). Data clustering (also termed modularity) has been used in the past as a classification tool for grouping of a set of given publications (Radicchi et al., 2004). In bibliographic networks, data clustering is employed to examine the local interconnections among nodes. This analysis can be used to understand if publications, directly connected to a given publication, cite with each other and if they tend to form local highly interconnected clusters of publications (Caschili et al., 2014). A high propensity to clustering might point out tight groups of publications belonging to the same research area or have similar research focus (Caschili et al., 2014). These help to gain an insight with regards to the current status of a research front such as DT-OSCM.

Gephi uses the Louvain algorithm as the default clustering tool, this is an iterative optimization model that aims to determine the optimal number of partitions that maximize the modularity index (Blondel et al., 2008). Modularity, which measures the density of links 
inside communities versus the links between communities, is gaining attention in the research community (Mishra et al., 2017). The modularity index of a partition is a scalar value between -1 and +1 that measures the density of links inside communities versus the links between communities (Fahimnia, 2015a). For a weighted network (i.e. networks with weighted links, such as the number of co-citing articles), the modularity index $Q$ can be calculated as:

$$
Q=\frac{1}{2 m} \sum_{a j}\left[A_{i j}-\frac{K_{i} K_{j}}{2 m}\right] \delta\left(C_{i}, C_{j}\right)
$$

where $A i j$ represents the weight of the edge between nodes $i$ and $j, k i$ is the sum of the weights of the edges attached to node $i\left(k_{i}=\sum_{j} A_{i j}\right), c i$ is the community to which node $i$ is assigned, $\delta(u, v)$ equals 1 if $u=v$; and equals 0 , otherwise, and finally $m=\frac{1}{2} \sum_{i j} A_{i j}$ (Blondel et al., 2008).

Eight clusters were created by the application of this algorithm to the 135-node bibliographic network in Gephi. The size of each cluster varied from 1 for cluster 6 to 44 for clusters 1 and 3 respectively. Clusters 5, 6, and 7 containing only 5, 1, and 4 articles respectively were dropped from further analysis as they did not appear to be significant to be considered as major literature clusters. This resulted in a 125-node network comprising of 5 clusters. Employing the partitioning function, a colour template was applied to the 125 -node network to distinguish between the clusters. Figure 4 illustrates the positioning of and interaction among the five clusters. An edge between two nodes indicates they have a at least one bibliographic coupling count, meaning that the two articles represented by the nodes have one or more articles they cite in common (Hosoya et al., 2017). The modularity index for this network is equal to 0.366 which indicates strong relationship between the nodes within each cluster and yet a relatively strong relationship between the nodes of different clusters. 


\subsubsection{Keywords co-occurrence analysis and research concepts identification}

To determine the area of research focus for each cluster we performed a keyword cooccurrence analysis. Keywords have the potential to effectively describe the contents of a paper (Rajagopal et al., 2017). Co-occurrence analysis is built on the assumption that a group of keywords could indicate the underlying themes and that the co-occurrences of keywords could reveal the association with the underlying themes ( $\mathrm{Hu}$ and Zhang, 2015). If two keywords occur simultaneously in an article, then they have a semantic relationship. The higher the co-occurrence frequency of two keywords, the greater is the correlation (Liu et al., 2012). Studies have used co-occurrence analysis to determine the knowledge structure in various research fields (Ravikumar et al., 2015; Stegmann \& Grohmann 2003).

In the first instance we extracted the keywords from all the 234 papers in our sample producing a list of 501 keywords. Table 10 shows the top 20 keywords by frequency of occurrence while Table 11 shows the top 20 keywords by eigenvector centrality. It comes as no surprise that the term "digital twin" ( $f=111, E V C=1)$ sits at the top of both tables. This also means that from the search terms employed, digital twin is by the far the most prominent term used in academic research. Most of the top keywords from both lists are either related to manufacturing or technology, this may infer as to the focus of most research in our sample. Indeed, the top co-occurring pair of keywords is digital twin and manufacturing $(f=55)$ as can be observed in Table 12, this is followed by digital twin and industry $4.0(f=37)$.

[Insert Table 10, 11, 12 near here]

We employed the eigenvector centrality measure to identify the 10 most influential papers (Fahimnia et al, 2015a; Hosoya et al., 2017) in each of the 5 literature clusters to identify the research themes. This is, with the exception of cluster 2 comprising of only 6 articles (see Table 13), inclusion of which was based on the strong interconnection between the articles, 
they all appear to be related to the same topic. VOS viewer (Van Eck and Waltman, 2013) was used to extract the keywords and perform the network mapping of keywords co-occurrence for each cluster. VOS viewer is a very useful tool for collecting bibliographical material, providing visualizations of the bibliographic connections of documents, journals, authors, as well as keywords by using a wide range of techniques including bibliographic coupling (Kessler, 1963) and co-occurrence analysis (Cancino et al., 2019).

[Insert Table 13 near here]

In VOS, network analysis is achieved by employing a unified framework that brings together the well-known technique of multidimensional scaling (van Eck et al., 2010) for mapping and a variant of modularity-based clustering (Newman and Girvan, 2004). This ensures that small clusters can always be identified by choosing a sufficiently large value for the resolution parameter (Waltman et al., 2010), making VOS viewer a powerful tool for analysing the keywords co-occurrence network of each literature cluster. Each cluster was labelled subjectively based on the popular keywords within the cluster.

The significance of a keyword can be assessed by its frequency count or total link strength (TLS) in VOS. In a keyword co-occurrence network the TLS attribute indicates the total strength of the co-occurrence links of a given keyword with other keywords (van Eck and Waltman, 2020). Thus, the more important and influential keywords tend to have higher TLS. VOS retrieved the keywords of all the papers in each cluster, the top 15 keywords for each literature cluster based on TLS are shown in Table 14. Figs. 6-10 represent the keywords cooccurrence networks of Clusters $1,2,3,4$, and 5 respectively. The sizes of the nodes are proportional to their weights measured in TLS, the higher the TLS of a keyword the larger the 
node. Lines between keywords represent links, the keywords central to the network with thicker edges denote the major research area of that cluster.

[Insert Table 14 near here]

\section{Discussion: Analysis of research clusters}

Analysis of the research clusters reveal broadly four value clusters and one enabler cluster. The value clusters include articles that help to illustrate how DT can be used to enhance supply chain activities at the level of supply chain processes as well as at the level of supply chain capabilities. The business processes value clusters are comprised of alternative manufacturing, smart manufacturing, product development and life-cycle management. In line with Croxton et al.'s (2001) categorisation of supply chain business processes it can be argued that alternative manufacturing and smart manufacturing are aligned with the manufacturing flow management process. According to Croxton et al. (2001), the manufacturing flow process is tasked with making the products and instilling the manufacturing flexibility needed to serve the target markets. The process comprises all activities necessary for managing the product flow through the manufacturing facilities and for obtaining, realising, and managing flexibility.

In a similar vein, arguably, product development and life-cycle management could be attributed to the product development and commercialisation process. Croxton and colleagues recognise the criticality of product development to the ongoing success of the firm. They thus view supply chain management as including integrating customers and suppliers into the product development process with the aim of reducing time to market. The discussions below begin to shine a light as to the potential of digital twin to optimise those supply chain business processes. 
Supply chain capability value cluster is made up of supply chain resilience and risk management. As such, the value proposition of digital twin is directed at a more abstract level as compared to the business processes discussed above. The enabler cluster is made of articles that broadly discuss technologies that can play a supportive role in the application of DT.

\subsection{Alternative manufacturing}

Cluster 1 is among the largest cluster comprising of 44 nodes and centrally located as can be observed in Figure 5. We have labelled this cluster as alternative manufacturing based on the most prominent keywords being additive manufacturing and remanufacturing. On close inspection of the co-occurrence network in Figure 6 we can also discerned the strong links between these terms and digital twin.

[Insert Figure 6 near here]

The field of additive manufacturing has seen an explosive growth in recent years due to renewed interest in manufacturing in the United States and other parts of the world (Bandyopadhyay and Bose, 2019). Additive manufacturing is a suite of emerging technologies that fabricates three-dimensional objects directly from digital models through an additive process, typically by depositing and "curing in place" successive layers of polymers, ceramics, or metals. Unlike traditional manufacturing processes involving subtraction (e.g., cutting and shearing) and forming (e.g., stamping, bending, and molding), additive manufacturing joins materials together to build products (Ford, 2014). These technologies have been widely researched and implemented to produce homogeneous and heterogeneous products with complex geometries (Butt, 2020). Properties and serviceability of additively manufactured components are affected by their geometry, microstructure and defects. These important 
attributes are now optimized by trial and error because the essential process variables cannot currently be selected from scientific principles (Knapp et al., 2017).

In this research stream authors explore how the digital twin technology could be leveraged to help reduce the flaws in the additive manufacturing process. For example, Gaikwad et al., (2020) combine predictions from a physical model with in-situ sensor signatures in a machine learning framework to show improved detection of flaws in the manufacturing of thin-wall titanium alloy part. Another way to eradicate flaws in the additive manufacturing process is through proactive maintenance. The research by Cahyati and Achdianto (2019) describes the prototyping of an additive manufacturing process with cyber-physical system enabling the creation of a virtual twin. In turn this allowed a virtual maintenance program to be made for the system.

A further issue associated with additive manufacturing besides defects is the slow manufacturing pace given that the filling of solid contours takes place with mere single deposition lines of material. In this context, Cai et al., (2020) demonstrate how a digital twin methodology could be developed to allow concurrent deposition of materials without collision among them. Similarly, Nagar et al., (2020) show how a digital twin system for additive manufacturing could be used to optimise the process through reduced downtime and larger productivity. As such, it could be argued that digital twin could be used to add value to varying processes resulting in improved speed, quality, efficiency, and productivity, these are key ingredients in achieving an effective and efficient manufacturing flow process for additive manufacturing.

Remanufacturing is the subject of the other subcluster. As an industrial process, remanufacturing concerns the restoration of products to useful life. It is often considered as 
an environmentally preferable choice of end-of-life option in comparison to material recycling or manufacturing new products (Sundin and Lee, 2012). However, the major problems in implementing a remanufacturing strategy result from the extreme uncertainty and variability of the remanufacturing environment (ljomah et al., 1999). Wang et al., (2020) also observe the lack of product multi-life-cycle remanufacturing process tracking management as particularly problematic. In this subcluster authors (e.g., Wang and Wang, 2019; Wang and Wang, 2020) approach solutions to the ongoing problems from a product lifecycle management perspective discussing different permutations as to how this is achievable by employing disruptive technologies such as cyber-physical systems and digital twins.

\subsection{Smart manufacturing}

Cluster 5 is the second smallest cluster comprising of 14 articles. Whilst the concept of smart manufacturing transcends all the clusters, it takes on a more prominent role in this cluster, Figure 7 displays a strong link between smart manufacturing and digital twin. Smart or intelligent manufacturing is an emerging form of production integrating manufacturing assets of today and tomorrow with sensors, computing platforms, communication technology, control, simulation, data intensive modelling and predictive engineering (Kusiak, 2018). The essence of smart manufacturing is the connection of all systems in the factory setting to create a federated digital twin and this would include logistics, supply chain, building services and other systems O'Sullivan et al. (2020). In this cluster authors investigate different permutations as to how the application of digital twin and cyber-physical systems could make manufacturing truly smart or intelligent.

[Insert Figure 7 near here] 
Research by Shahidi et al. (2021), Tan et al. (2019) and Zhang et al. (2019) focus on smart factories. Shahidi and colleagues discuss the enablers of smart factories particularly focusing on a big data architecture for digital twin application. They come up with the concept of decentralized Digital Shadows (dDS) which is essentially a digital architecture which promises optimal space allocation for big data and fast response to modelling. Tan and colleagues are also concerned with data integration in a smart factory digital twin approach proposing a digital twin construction framework and scheme for inputting data derived from the internet of things (loT) into a simulation model. Zhang and colleagues are primarily interested in how to realize the interconnection and interoperability of shop-floor elements between the physical space and the virtual space. As such, the main focus of this paper concerns the implementation approach of digital twin-driven cyber physical production system (CPPS) towards smart shop floor particularly for the production phase. To achieve this the authors propose and validates a digital architecture solution.

Works by Söderberg et al. (2017) and Schleich (2017) examine the issue of quality assurance in the context of smart manufacturing. Söderberg and colleagues' paper specifies and highlights functionality and data models in a digital twin environment necessary for real-time geometry assurance for production systems. Schleich and colleagues recognise the need for more realistic virtual models of manufactured products in order to bridge, what they assessed as a gap between design and manufacturing. As such, they propose a comprehensive reference model based on the concept of Skin Model Shapes, which serves as a digital twin of the physical product in design and manufacturing. Such a framework could also be put to use to address quality issues in production and design. Measuring performance is an important element of the manufacturing flow process. Croxton et al. (2001) argue that at the core of performance management includes the analysis of product quality and an examination of the 
root causes of quality problems. In this light, it can be argued that a digital twin solution could add significant value to the manufacturing flow process.

\subsection{Product development and lifecycle management}

Cluster 3 is the joint largest community also comprising of 44 articles. As can be observed in Table 14, most of the top 15 keywords are related to processes involving product such as complex product, product life cycle management and product optimisation, thus product development and lifecycle management was deemed a suitable name to represent this cluster. Figure 8 illustrates the key linkages in this cluster resonating the statistics in Table 14. In particular, complex product, data management, smart product, and product lifecycle management have the strongest link with digital twin. Careful inspection of the articles does reveal the presence of three subclusters comprising of complex product, smart product, and product lifecycle management (PLM) with data management playing an integral part in these processes.

[Insert Figure 8 near here]

A complex product is one that has many constituent parts, each part is manufactured to high precision backed up by a level of research and development, for example, a computer server, sports car, and refrigerator. These are normally innovative products with less predictable demand (Wong et al., 2002). Zhuan et al. (2020) observe that the assembly process for these complex products involves high complexity, strong dynamics, many uncertainties, and frequent rework and repair, especially in the model development stage. According to Sun et al. (2020), the traditional assembly process of complex products is based on manual experience, which results in low assembly efficiency and poor-quality consistency. The studies in this subgroup variously recognise the potential of cyber-physical system to address the 
assembly problems of complex products. Consequently, each of the three papers proposes a digital twin-driven assembly-commissioning approach for complex products. Case studies involving the assembly of a satellite (Yi et al., 2020) and an electro-hydraulic servo valve (Sun et al., 2020) show the effectiveness of the respective approaches.

Gutiérrez et al. (2013, p. 206) define a smart product as "an autonomous object which is designed for self-organised embedding into different environments in the course of its lifecycle and which allows for a natural product-to-human interaction. Smart products are able to proactively approach the user by using sensing, input, and output capabilities of the environment thus being self-situational, and context-aware." The two papers in the subcluster focus on smart product development and innovation, two areas adjudged as requiring attention. In Lin et al (2021), evolutionary digital twin (EDT) is proposed as a new mode for intelligent industrial product development. A case study shows that EDT instils more flexibility and adaptability in the development process of intelligent industrial product. Beyond intelligent products, flexibility and adaptability are increasingly needed in more general product development particularly given the uncertain nature of demand and the move away from mass customisation toward personalisation. By instilling such attributes in the product development process the value-added potential of DT is revealed.

Zheng et al. (2018) on the other hand applies the digital twin concept to the service innovation of smart product which they refer to as smart product-service systems (PSS). The service innovation is enabled by a platform-based approach and generated in a data-driven manner. A case study involving a personalised wearable is used to validate the digital twin framework for service innovation. 
Optimisation of the process of product lifecycle management (PLM) is an increasingly important objective for manufacturing enterprises to improve their sustainable competitive advantage (Zhang et al. 2017). PLM is often defined as a set of functions and procedures which allows the management and exploitation of the data at the same time defining the products and the processes implemented for their developments (Zina, 2006). Huang et al. (2020) note that in PLM, there are many participating actors constructing a complicated network with enormous product lifecycle data, this makes the implementation of a PLM solution difficult. The papers in this subcluster discuss the various ways digital twin could offer a workable solution to the data management issues faced by PLM. Huang et al. (2020) discuss a data management method for digital twin of PLM based on blockchain technology. It is argued that blockchain technology enhances data sharing efficiency among participating actors. The effectiveness of the proposed data management method is evaluated using a case study which shows that the proposed framework can solve the data management problem.

\subsection{Supply chain resilience and risk management}

Cluster 2, which we have named supply chain resilience and risk management, is the smallest comprising of only 6 articles. From Figure 5, it can be observed that this community is somewhat removed from the core of the network. Nevertheless, the articles form a tightly woven literature cluster focussing on examining the role of cyber-physical systems and digital twin in the development of supply chain resilience. Figure 9 indeed reveals the strongest links in this cluster are between supply chain, resilience, digital twin, and ripple effect. These studies are inspired by the ongoing Covid-19 pandemic, conceptualised as the single most pervasive source of supply chain disruption risk in most of the studies. As such, the studies in this cluster mostly focus on discussing the digital strategies built around cyber-physical 
systems and digital twins to mitigate supply chain disruptions and recover in case of severe disruptions (Ivanov and Dolgui, 2019).

[Insert Figure 9 near here]

Predicting the impact of epidemic outbreaks on global supply chains is the subject of Ivano's (2020) study. Supply chains are complex systems, unlike manufacturing or a piece of machinery where the avatar is a virtual representation of the physical object, the conceptualisation of a supply chain digital twin requires the modelling of the supply chain design supported by real time operational parameters (Ivanov and Dolgui, 2020) employing both prescriptive and predictive analytics. In this study the value-added potential of a digital twin is thus conceptualised at the level of supply chain capability. Previous research has positioned supply chain resilience as a dynamic capability to prepare for unavoidable risk events and to respond to and recover from unexpected disruptions. Ivanov and Dolgui show how the application of a DT could enable such a dynamic capability by matching supply chain designs to different sets of disruption patterns while key performance measures are monitored. Decision makers can draw on the outcomes of these predictive simulations and prescriptive optimization to transform the supply chain design to absorb the negative effects from a range of different risk sources (Teece, 2007) in real time. Ivano's study for instance, reveal that the timing of the closing and opening of the facilities at different echelons might be a significant factor that determines the epidemic outbreak impact on the SC performance rather than an upstream disruption duration or the speed of epidemic propagation.

Three studies in this cluster recognise the ripple effect (Ivanov and Dolgui, 2019; Ivanov et al., 2019; Ivanov and Dolgui, 2020) as a significant threat to supply chain resilience resulting from disruptions. The ripple effect occurs when a disruption, rather than remaining localized or 
being contained to one part of the supply chain, cascades downstream and impacts the performance of the supply chain (Ivanov and Dolgui, 2019). Ripple effect control is thus seen as a critical risk management task to achieve supply chain resilience. In these studies a supply chain digital twin for risk management is conceptualised and the ripple effect of an epidemic outbreak in global supply chains is modelled to evaluate optimal supply chain risk measures and potential recovery paths. Based on the results of simulations, redundancy, flexibility, and leanness are assessed as key supply chain capabilities effective to control the ripple effect caused by a global pandemic. Similarly, these studies are examples of value-creation at the level of supply chain capability level orchestrated by the application of a DT.

\subsection{Enabling technologies}

Cluster 4 is dedicated to enabling technologies particularly focussing on blockchain as can be observed in Figure 10. Disruptive technologies are foundational to the digital twin concept, they are the very bedrock enabling the physical-virtual manifestations. Some of the core disruptive technologies captured across the five clusters include cyber physical system, industry 4.0, internet of things, robotics, artificial intelligence, virtual reality, simulations, 3D printing, machine learning, big data analytics, predictive analytics, smart contract and of course blockchain. Figure 10 shows a strong link between digital twin, blockchain and smart contracts. Blockchain and smart contract are related concepts. Blockchain is defined as a distributed data base or digital ledger that records transactions of value using a cryptographic signature that is inherently resistant to modification (Mylrea, and Gourisetti, 2017). Applying blockchain based smart contracts presents an opportunity to increase the speed, scale, and security of transactions.

[Insert Figure 10 near here] 
The articles in this cluster investigate how blockchain technologies could be dispensed to enhance the existing digital twin frameworks. Hasan et al. (2020) point out some limitations of current approaches, systems and technologies of digital twin related to centralization and falling short of providing trusted data provenance, audit, and traceability. A blockchain approach to digital twin promises to resolve some of those limitations, possessing the advantage of distributed consensus and tampering-resistant, blockchain makes decentralized and collaboration in manufacturing possible (Leng et al., 2019). Hasan and colleagues propose a blockchain-based creation process of digital twins to ensure secure and trusted traceability, accessibility, and immutability of transactions, logs, and data provenance. Their approach uses smart contracts to govern and track transactions initiated by participants involved in the creation of digital twins.

To address the challenge of ensuring trust among makers in the social manufacturing paradigm, Leng et al. (2019) propose a blockchain-driven decentralized self-organizing model they named Makerchain, through which a decentralized network of makers can cooperate in a manufacturing ecosystem. The implementation of the Makerchain is validated via a case study of 3D printer manufacturing based on an open- source project named RepRap. The work by Leng et al. (2020) addresses the problem of inflexibility of centralized control associated with industrial internet of things (IloT) with respect to the manufacturing of individualized products. They argue that the current system is unable to cope with the disturbance and changes required of individualized manufacturing. As such, they propose a novel iterative bilevel hybrid intelligence model they called ManuChain to get rid of unbalance/inconsistency between holistic planning and local execution in individualized manufacturing systems. ManuChain is based on incorporation of blockchain into a digital twin for individualized manufacturing on a decentralized network. A blockchain data model and smart contracts are 
built to autonomously execute plans being given to the manufacturing unit based on realtime tracking of industrial internet of things (IloT) events. Zhang et al. (2020) also employ a blockchain approach to address the problem of inflexibility of IloT in the intelligent manufacturing paradigm. To address the problem, the authors combine IloT with the permissioned blockchain and propose a novel manufacturing blockchain of things (MBCoT) architecture for the configuration of a secure, traceable, and decentralized intelligent manufacturing system (IMS).

\section{Conclusion}

Digital twin promises to revolutionise the practice of operations and supply chain management and is the most recent in a long list of technologies that have helped give strategic purchase to the field. The practice-based literature reports on the marvels of this technology accounting for its value-added potential in glorious details. Given the preeminence of BDA in the functioning of a DT, in this work we have relied on the literature on BDA-enabled capabilities to advance arguments to support the use of DT in OSCM as a dynamic capability and by doing so recognising its ability to create value is incumbent on its embeddedness in supply chain processes. We have then examined the literature to capture how far the conversation on the value potential of DT in OSCM has reached in the academic world.

Our findings reveal that while this conversation is still in its infancy value clusters are appearing in the literature. Researchers have examined the value-added potential of DT at the level of supply chain business processes (Croxton et al., 2001) with an accentuated focus on manufacturing flow management and product development and commercialisation. We believe that these are maturing literature streams. Furthermore, there is an emerging cluster 
which focusses attention on value at a more abstract capability level. This emerging cluster discusses supply chain resilience as a dynamic capability and the pivotal role DT can play in its enablement. These findings provide an initial point of entry for discussions with respect to practice, theory development, and future research.

\subsection{Implication for practice}

Our analysis of the literature reveals the huge potential of DT to add value to the manufacturing flow process and the product development process. With respect to the manufacturing flow process the findings reveal that DT adds value mostly through process optimization (Cimino et al., 2019; Sun et al., 2020; Leng et al.,2020) and predictive maintenance (Cahyati et al., 2019; Cimino et al., 2019). Manufacturing in its various forms has frequently been accused of being wasteful often to the detriment of the environment. The findings in this review suggest avenues where DT could be employed as an operational capability to streamline processes, remove manufacturing flaws, enhance quality and efficiency, and improve the overall productivity. Although evidence linking these value improvements to value manifestations such as waste reduction, cost reduction and overall firm performance is not provided, these appear to be the natural conclusion.

There has been much talk by industrial nations such as the US and the UK, regarding the reshoring of manufacturing activities. In the context of the UK, Brexit has provided the momentum for the made in Britain movement. Much of the manufacturing activities were offshored from the UK in the 1980s (Bailey and De Propris, 2014) under the leadership of Margaret Thatcher citing the growing lack of competitiveness of the UK as the key economic argument. Digital twin potentially helps to redress this situation and could be seen as the raison d'etre to fuel the reshoring or made in Britain ambitions. The UK has indeed recognised 
the benefits of DT to manufacturing and supports numerous initiatives (e.g., Slingshot Simulations). As such our findings serve to reinforce the need for a national debate on the benefits of DT as a distinct manufacturing capability as well as the development of inclusive industrial policy framework that sets out mechanisms to support and encourage UK industries, especially SMEs, to adopt such digital technologies.

Our findings also provide some points of reflections as to how DT could add value to the product development process particularly with respect to innovation. An interesting emphasis concerns product development from the perspective of whole lifecycle management which also tend to carry an environmental sustainability connotation. This is a sector which is gaining popularity especially amongst millennials believed to be more environmentally conscious than previous generations. Our findings reveal two different facets of DT as a dynamic capability, it can be leveraged into a dynamic collaborative capability for the large number of actors involved in the product development process as well as dynamic capability for information processing for product life-cycle data handling. Manufacturing firms can thus employ DT to help reconfigure existing capabilities and achieve competitive advantage. However, this comes with a caveat concerning data management and the issue of trust is also at play. While trust can be nurtured data management issues such as data quality, privacy and security are a different beast altogether. Employing a DT in the context of product development potentially involves the collection of large amounts of personal data. While legislation exists (such as the General Data Protection Regulation (GDPR)) aimed at safeguarding personal data, there is a lack of a legal framework as to the use of product data collected from embedded technologies for commercialisation purposes. This is a grey area that needs addressing from a legal perspective. Companies wishing to develop a DT solution 
to build dynamic capabilities around the product development process would do well to consider such issues very carefully to avoid potential costly legal consequences in the future.

The dynamic capability potential of DT is also visible when firms can leverage it in order to reconfigure supply chain design in anticipation of expected disturbances. The findings reveal how firms can employ DT to develop resilience in their supply chains. Resilience is an important supply chain dynamic capability that can help firms survive unexpected events and is particularly salient in times of a global health crisis such as Covid-19. Whether a supply chain is resilient or not can often determine whether a firm survives or not. Therefore, our findings provide a lens to understand how firms could instil survivability in their operations by employing DT solutions to create dynamic capabilities. We also wish to sound a cautious note here to companies thinking about developing a DT solution for supply chain resilience. Whilst such a system utilises real-time data from operational processes, a large amount of data is third party data, and this may be data held by private entities or public bodies both local and foreign. This leads to questioning the issues of data acquisition and data quality. These are issues beyond the control of firms but nevertheless must be factored in from the onset of an investment project to develop a DT for supply chain resilience. A potential solution could be to outsource data acquisition to third party data companies; however, the veracity of such data remains a sticking point. Furthermore, firms would need to ensure an effective internal data management system to support such an initiative and with this comes the prerequisite IT infrastructure.

\subsection{Implication for theory development}

We have pursued the analysis of extant DT-OSCM literature on the conceptual assumption that DT could be treated as an enabler of dynamic capability in supply chains. As pointed out, 
the literature is still in its infancy and DT is yet to be theoretically conceptualised as a valuecreation vehicle for OSCM in any meaningful way. Our findings do however show that when embedded in existing processes DT has the ability to improve and at times transform them into higher level capabilities. Furthermore, dynamic capabilities have been described as a bundle of unique resources and capabilities (Wang and Ahmed, 2007). DT is a constellation of different technologies, and these are increasingly ubiquitous. Therefore, it can be argued that the value potential of DT also rests in the unique way companies bundle these technologies to create a distinctive DT architecture. As such, we believe that our analysis of the literature is foundational to initiating a conversation about the conceptual framing of DT in OSCM. There might also be a need to examine the value creating mechanisms at a more granular level where the microfoundations of capabilities reside. This will necessarily require conceptualisations at lower level of abstractions to capture the mechanisms of interaction between DT and supply chain processes. As such, in the tradition of IT-enabled supply chain management, we believe that DT will find a home under the auspices of the resource-based view.

\subsection{Implications for future research}

The findings and conclusions of this review have some important implications for future research. Supply chain resilience and risk management is viewed as an emerging research stream although resilience and risk have a long tradition in quantitative and modelling approaches. Supply chain practitioners are particularly well placed to benefit from insights from carefully crafted research on the benefit of a supply chain digital twin to inform robust and adaptable supply chain design. Digital twin is fast transcending the manufacturing landscape, this presents many opportunities for empirical work particularly in the shape of 
case studies that could produce actual knowledge about the specificities, challenges, and benefit of a digital twin approach to supply chain resilience and risk management.

It is disappointing to note the limited contribution of supply chain and operations management scholars to the field so far. However, on the positive side this presents a great opportunity for those scholars to make significant contributions towards the development of the field. For example, there is a pressing need to address the first and last mile issues in supply chain, we see supply chain digital twin as offering useful insights as to how to optimise decision making regarding these functions. On the whole, we believe future work could examine the mechanics of value creation where DT intersects with supply chain business processes such as demand management, order fulfilment, supply management, and customer services management.

We further see the need for the development of a scale enabling the measurement of the "digital twin capability" construct. This will enable the conceptualisation of digital twin as a capability or dynamic capability in empirical work by drawing on the resource-based view. Research work employing survey instruments could test the relationship between digital twin capability and value manifestations such as firm performance and customer satisfaction. Furthermore, this will also allow antecedents or enablers of digital twin capability to be closely examined. These developments would help move the field along and attract a more diverse pool of scholars.

As we have already highlighted, theoretical work in this area also remains outstanding, we urge scholars to take on the challenge, this can be in the form of conceptual work or case studies as directed above. Digital twin presents the supply chain with endless possibilities to achieve optimal functioning, we have highlighted a few areas where we think supply chain 
research would benefit the most. However, a digital twin approach has the potential to revolutionize operations and supply chain management research we hope the findings in this work can provide the momentum to make this happen.

\subsection{Limitations}

In spite of our best efforts to ensure robustness and inclusivity, our research is limited by the exclusive focus on peer reviewed material. This could be addressed by future research that employs non-academic publications as well as a broader swathe of academic articles. In addition to the areas of research identified above, future empirical research could take a more contextual approach with the aim of understanding the mechanics of DT-OSCM across different economic sectors. Another limitation concerns the use of only one database to extract the articles for this review. Although SCOPUS is by far the most comprehensive and updated repository for scholarly works, there is a chance that our review may have missed studies published in other outlets.

\section{References}

